\title{
Correction to "Systems Pharmacological Analysis of Paclitaxel-Mediated Tumor Priming that Enhances Nanocarrier Deposition and Efficacy"
}

In reviewing the above article [Ait-Oudhia S, Straubinger RM, and Mager DE (2013) $J$ Pharmacol Exp Ther 344(1): 103-112; DOI: https://doi.org/10.1124/jpet.112.199109], the authors discovered panels $\mathrm{A}$ and $\mathrm{E}$ in Figure 2 are incorrect, along with typos in several parameters (mostly units) in Table 2 . The modeling and simulations have been faithfully reproduced, and these errors do not change the results and conclusions of the paper. The correct versions of Table 2 and Figure 2 appear below and in the PDF and HTML versions of this article.

The authors apologize for any inconvenience caused by these errors.

TABLE 2

Pharmacokinetic and pharmacodynamic parameters and coefficients of variations (\%CV)

\begin{tabular}{|c|c|c|}
\hline Parameter (unit) & Definition & Estimate (\%CV) \\
\hline \multicolumn{3}{|l|}{ Pharmacokinetics } \\
\hline $\mathrm{CL}_{\mathrm{pac}}\left(10^{-1} \mathrm{~L} / \mathrm{h} / \mathrm{kg}\right)$ & PAC linear clearance & $0.85(24.9)$ \\
\hline $\mathrm{CL}_{1}(\mathrm{~L} / \mathrm{h} / \mathrm{kg})$ & PAC clearance for peripheral compartment 1 & $0.38(17.3)$ \\
\hline $\mathrm{CL}_{2}(\mathrm{~L} / \mathrm{h} / \mathrm{kg})$ & PAC clearance for peripheral compartment 2 & $0.24(31.4)$ \\
\hline $\mathrm{V}_{\mathrm{pac}}\left(10^{-1} \mathrm{~L} / \mathrm{kg}\right)$ & PAC central volume of distribution & $0.82(47.8)$ \\
\hline $\mathrm{V}_{\text {p1-pac }}\left(10^{4} \mathrm{~L} / \mathrm{kg}\right)$ & PAC volume for peripheral compartment 1 & $1.8(54.3)$ \\
\hline $\mathrm{V}_{\mathrm{p} 2-\mathrm{pac}}(\mathrm{L} / \mathrm{kg})$ & PAC volume for peripheral compartment 2 & $1.5(17.4)$ \\
\hline $\mathrm{CL}_{\text {cre }}\left(10^{-1} \mathrm{~L} / \mathrm{h} / \mathrm{kg}\right)$ & Cremophor ${ }^{\circledR}$ EL clearance & $0.79(40)$ \\
\hline $\mathrm{V}_{\text {cre }}\left(10^{-1} \mathrm{~L} / \mathrm{kg}\right)$ & Cremophor $^{\circledR}$ EL volume of distribution & $0.5(49.1)$ \\
\hline $\mathrm{k}_{\mathrm{rel}}(\mathrm{h}-1)$ & Release rate constant for SSL-DXR & $0.046(2.8)$ \\
\hline $\mathrm{CL}_{\mathrm{DXR}}\left(10^{-1} \mathrm{~L} / \mathrm{h} / \mathrm{kg}\right)$ & SSL-DXR linear clearance & $0.42(11.2)$ \\
\hline $\mathrm{k}_{\mathrm{on}}\left(10^{-2} \mathrm{~h} \cdot \mu \mathrm{g} / \mathrm{mL}\right)^{-1}$ & Second-order binding affinity constant for PAC & $5.15(25.3)$ \\
\hline $\mathrm{k}_{\text {off }}\left(10^{-2} \mathrm{~h}^{-1}\right)$ & First-order dissociation constant for PAC & $4.09(141)$ \\
\hline CLD (L/h/kg) & SSL-DXR clearance for peripheral compartment & $0.85(39.8)$ \\
\hline $\mathrm{V}_{\text {DXR }}\left(10^{-1} \mathrm{~L} / \mathrm{kg}\right)$ & SSL-DXR central volume of distribution & $0.7(39.8)$ \\
\hline $\mathrm{V}_{\mathrm{p} \_\mathrm{DXR}}\left(10^{-1} \mathrm{~L} / \mathrm{kg}\right)$ & SSL-DXR volume for peripheral compartment & $1.06(39.8)$ \\
\hline $\mathrm{Q}_{\mathrm{T}}(\mathrm{mL} / \mathrm{h})$ & Tumor blood flow & $3.38(7.36)$ \\
\hline $\mathrm{Kp}_{\mathrm{PAC}}$ & Partition coefficient for PAC & $0.044(24.8)$ \\
\hline Kp_DXR & Partition coefficient for SSL-DXR & $0.085(7.6)$ \\
\hline $\mathrm{B}_{\mathrm{p}}$ & Linear binding of PAC to plasma proteins & $21^{\mathrm{a}}$ \\
\hline$B_{\text {sat }}$ & Saturable binding of PAC to plasma proteins & $35^{\mathrm{a}}$ \\
\hline $\mathrm{k}_{\mathrm{d}}(\mu \mathrm{g} / \mathrm{mL})$ & Dissociation constant from plasma proteins & $4.38^{\mathrm{a}}$ \\
\hline $\mathrm{fb}_{\mathrm{DXR}}(\%)$ & Fraction of doxorubicin bound to plasma proteins & $85^{\mathrm{b}}$ \\
\hline \multicolumn{3}{|l|}{ Pharmacodynamics } \\
\hline $\mathrm{E}_{\max -\mathrm{PAC}}(\%$ apoptosis$)$ & Maximum effect for PAC apoptotic signal & $18.04(10.01)$ \\
\hline $\mathrm{EC}_{50-\mathrm{PAC}}(\mu \mathrm{g} / \mathrm{mL})$ & Cre-pac concentration inducing $50 \%$ of $\mathrm{E}_{\text {max-pac }}$ & $7.217(12.7)$ \\
\hline$\gamma$ & Hill coefficient for Cre-pac & $2.22(45.7)$ \\
\hline $\mathrm{E}_{\text {max-DXR }}(\%$ apoptosis $)$ & Maximum effect for SSL-DXR apoptotic signal & $70.59(3.08)$ \\
\hline $\mathrm{EC}_{50-\mathrm{DXR}}(\mathrm{nM})$ & SSL-DXR concentration inducing $50 \%$ of $\mathrm{E}_{\max -\mathrm{DXR}}$ & $14.34(5.24)$ \\
\hline$\tau_{\text {Apo-PAC }}(\mathrm{h})$ & Single compartment mean transit time for PAC & $9.14(42.6)$ \\
\hline$\tau_{\text {Apo-DXR }}(\mathrm{h})$ & Single compartment mean transit time for SSL-DXR & $5.273(4.45)$ \\
\hline $\mathrm{K}_{\mathrm{g}}\left(10^{-2}, \mathrm{~h}^{-1}\right)$ & Net growth rate constant & $0.565(2.55)$ \\
\hline $\mathrm{R}_{\mathrm{ss}}(\%$ change $)$ & Maximum unperturbed \% change in tumor size & $1857(7.3)$ \\
\hline$\tau_{d}(h)$ & Time to death & 22.59 (13.7) \\
\hline$\alpha(1 / \%$ apoptosis $)$ & Slope of PAC concentration-dependence of apoptosis induction & $4.31(15.2)$ \\
\hline$\beta$ (1/\% apoptosis) & Slope of SSL-DXR concentration-dependence of apoptosis induction & $0.93(21.5)$ \\
\hline$\theta$ (1/\%apoptosis) & Coefficient to $\mathrm{K}_{\mathrm{p} \_\mathrm{DXR}}$ after tumor priming & $0.27(38.4)$ \\
\hline
\end{tabular}

${ }^{a} B_{P}, B_{s a t, P}$, and $k_{d}$ parameters were fixed from (Bulitta et al., 2009).

$\mathrm{bfb}_{\text {DXR }}$ parameter was fixed from (Danesi et al., 2002) 
A

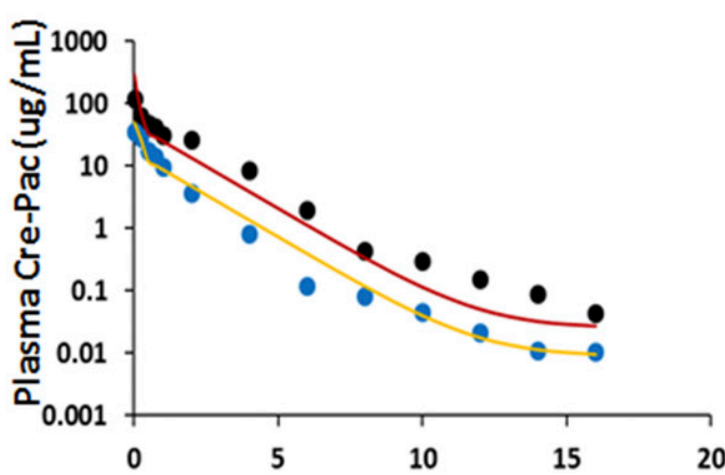

C

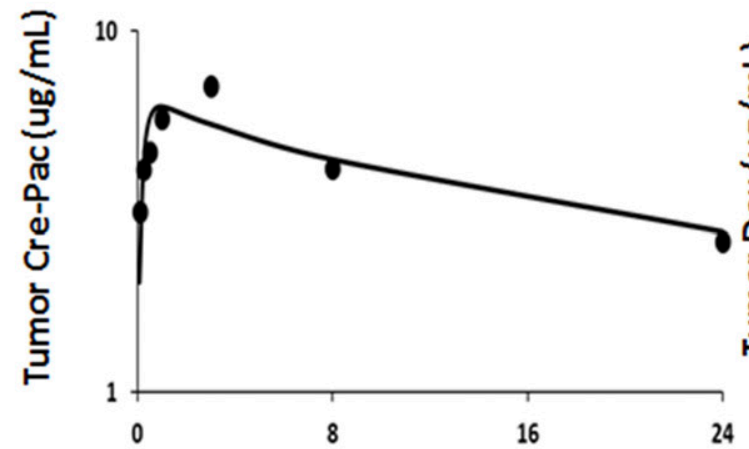

B

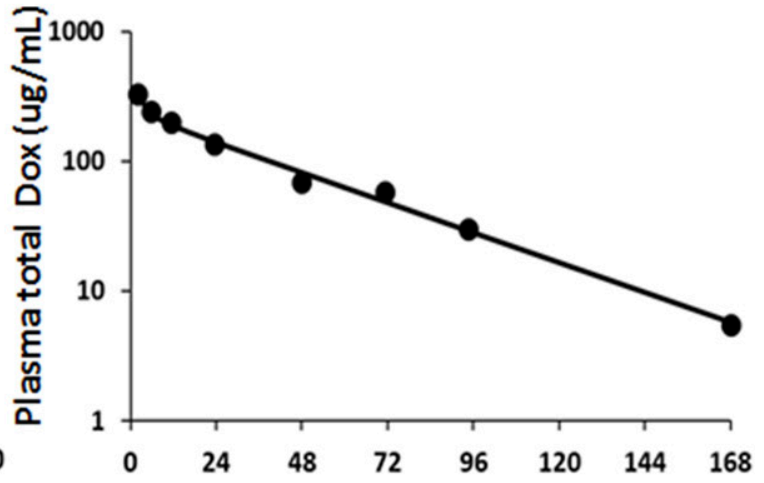

D

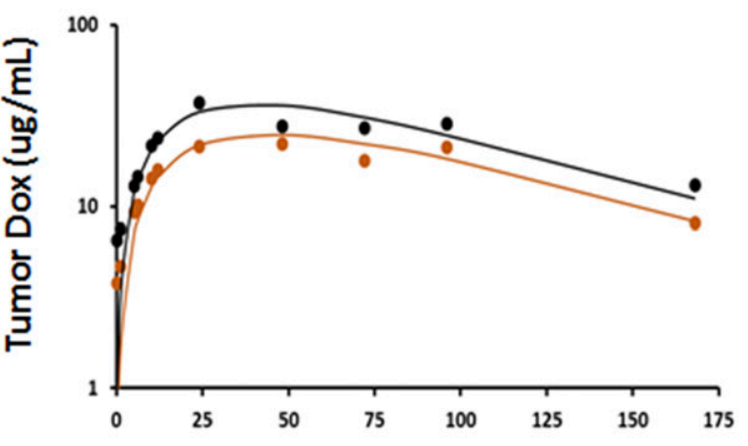

E

F

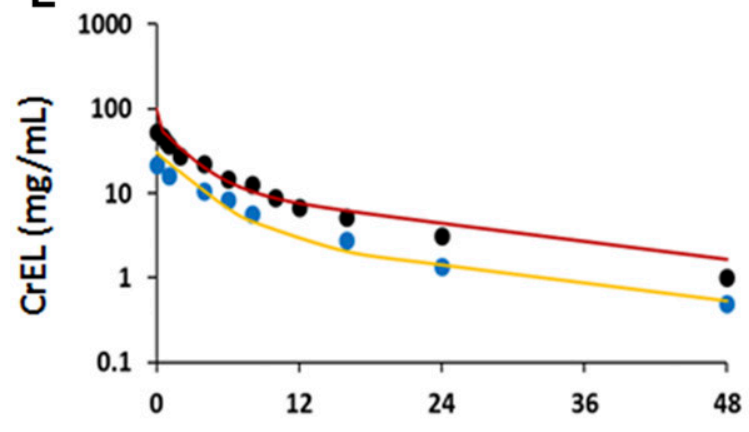

\section{Time (h)}

Figure 2: Pharmacokinetics of Cre-pac, CreEL, and SSL-DXR in plasma and tumor. In all panels, symbols represent observed data and lines represent model predictions. (A) PAC plasma concentrations (Sparreboom et al. (1996b)) for the two largest doses, (B) total plasma concentrations of DXR after SSL-DXR administration (Lu et al., 2007). (C) PAC tumor concentrations (Desai et al., 2006). (D) Total tumor DXR concentrations after administration of SSL-DXR alone (orange) or SSL-DXR administered $48 \mathrm{~h}$ after dosing with Cre-pac (black) (Lu et al., 2007). (E) CreEL plasma concentrations (Sparreboom et al. (1996b)) for the two largest doses. (F) free plasma concentrations of DXR (Xiong et al. (2005)). 